\title{
Size-Dependent Specific Surface Area of Nanoporous Film Assembled by Core-Shell Iron Nanoclusters
}

\author{
Jiji Antony, ${ }^{1}$ Joseph Nutting, ${ }^{1}$ Donald R. Baer, ${ }^{2}$ Daniel Meyer, ${ }^{1}$ Amit Sharma, ${ }^{1}$ and You Qiang ${ }^{1}$ \\ ${ }^{1}$ Department of Physics, University of Idaho, Moscow, ID 83844, USA \\ ${ }^{2}$ EMSL, Pacific Northwest National Laboratory, Richland, WA 99352, USA
}

Received 31 January 2006; Revised 22 August 2006; Accepted 28 August 2006

Nanoporous films of core-shell iron nanoclusters have improved possibilities for remediation, chemical reactivity rate, and environmentally favorable reaction pathways. Conventional methods often have difficulties to yield stable monodispersed core-shell nanoparticles. We produced core-shell nanoclusters by a cluster source that utilizes combination of Fe target sputtering along with gas aggregations in an inert atmosphere at $7^{\circ} \mathrm{C}$. Sizes of core-shell iron-iron oxide nanoclusters are observed with transmission electron microscopy (TEM). The specific surface areas of the porous films obtained from Brunauer-Emmett-Teller (BET) process are size-dependent and compared with the calculated data.

Copyright (C) 2006 Jiji Antony et al. This is an open access article distributed under the Creative Commons Attribution License, which permits unrestricted use, distribution, and reproduction in any medium, provided the original work is properly cited.

\section{INTRODUCTION}

Iron nanoparticles are interesting due to their smaller size and higher reaction efficiency. Nanoporous structures are a branch of nanomaterials that have significant importance due to their ability to absorb and react, as they possess large surface area. Nanoporous materials have applications as catalysts, sensors, electrodes for batteries and fuel cells $[1,2]$. Zero-valent iron $[\mathrm{Fe}(0)]$ nanoparticles react more quickly than their oxides and are of great importance due to their high oxidation capability and rapid kinetics in addition to their ability to reach the deep sites of contamination [3]. The iron-iron oxide core-shell nanoparticles can dechlorinate chlorinated hydrocarbons, a major source of environmental contaminant. The oxide shell provides a passivating layer that imparts stability to the particles in aqueous suspension [3] by preventing further oxidation, so that the particles react slowly for extended period. The iron nanoparticles can react with contaminants in the environment up to 8 weeks and can flow along with groundwater for a distance of $20 \mathrm{~m}$ [4]. Rapid in situ reduction of trichloroethelyne is observed using nano-iron. Up to $99 \%$ of the trichloroethelyne is reduced within a few days [4]. Potentially important aspect of nanoparticle structure, including protective shell or impurities, is to alter the final products formed during the oxidation of chlorinated hydrocarbons to form environmental friendly products [5]. Understanding the chemical properties of nanoparticles can be achieved by controlling the cluster structure and their shells. Control over the iron nanoparticles by conventional methods is difficult. The knowledge of the cluster components and their interaction mechanisms with gaseous or solution environments is an important requirement to understand the cluster.

Here we report the synthesis and characterization of nanoporous, nanostructured cluster film of iron along with the study of size-dependent enhancement of specific surface area (SSA).

\section{EXPERIMENTAL DETAILS}

Core-shell iron nanoclusters were synthesized from a $75 \mathrm{~mm}$ diameter iron target [6-8] of $3 \mathrm{~mm}$ thickness. Target acts as the cathode of the sputtering gun inside the third generation nanocluster source. The schematic representation is given in Figure 1.

Aggregation tube cooled by chilly water promotes the cluster synthesis at low temperatures ranging from $3^{\circ} \mathrm{C}$ to room temperature. In this experiment, the clusters were prepared at $7^{\circ} \mathrm{C}$. Inert gases are present inside the aggregation chamber, which acts as carrier gases, and Ar causes the sputtering. DC power when applied to the sputtering gun causes the gaseous Ar near the target to ionize and this generates $\mathrm{Ar}^{+}$ions. $\mathrm{Ar}^{+}$ions sputter the iron atoms from the surface of the target into the aggregation chamber where they aggregate due to low temperature and form clusters. Clusters move from one chamber to the other due to the gas flow and 


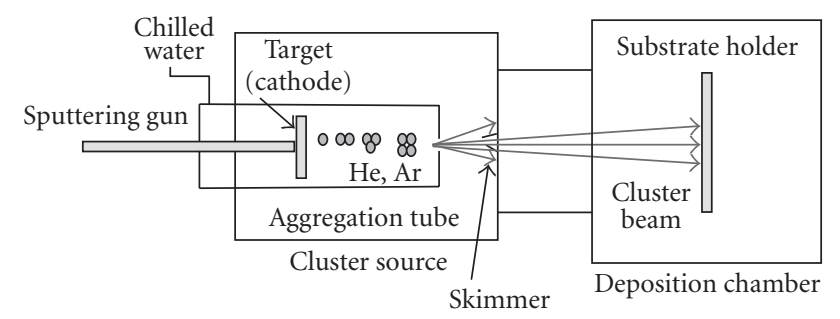

FIgURE 1: Schematic representation of the third generation nanocluster source.

pressure differences between the chambers and reach deposition chamber. Here the nanoclusters are deposited on substrates like TEM grids or Si wafers placed on the substrate holder. Size of the iron-iron oxide core-shell nanoclusters is varied using inert gas ratio, pressure, growth distance, and power supplied to the gun. Detailed information about the preparation technique can be found in [6-12]. To confirm our understanding of the properties of these particles, a comparison is made between the microscopic measurements of particle structures using transmission electron microscopy (TEM) and atomic force microscopy (AFM) and the surface area (SA) analysis using BET. Formation of well-defined particles provides useful materials for the study of chemical properties of nanoparticles.

\section{RESULTS AND DISCUSSION}

Low-resolution TEM image of monodispersed iron-iron oxide core-shell nanoclusters of $\sim 21 \mathrm{~nm}$ size exposed to atmosphere is shown in Figure 2(a). Figure 2(b) is a highresolution TEM image of a core-shell nanocluster. Thickness of the pure $\mathrm{Fe}(0)$ core and the iron oxide shell of this coreshell structure can be clearly seen from the high-resolution TEM image. The oxide shell measured with high-resolution TEM shows a thickness of $2-3 \mathrm{~nm}$, and is very stable. The characterizations of $\mathrm{Fe}(0)$ core and oxide shells are reported elsewhere [7]. TEM images show core-shell structure for particles exposed to the air for several months, which reveals the stability of iron-iron oxide core-shell nanoclusters.

The porous nature of the cluster film, observed from the tapping mode AFM data is given in Figure 3. Here we see the cluster film deposited on Si substrate. The image below is in $2 \mu \mathrm{m}$ range along $x-y$ direction and $20 \mathrm{~nm}$ in $z$-direction. The image RMS value is $1.98 \mathrm{~nm}$. As the deposition takes place in low energy range, the clusters retain their original shapes. The randomly deposited nanoclusters give porous nature to the film.

SSA is the SA per mass. Mathematically, SSA can be calculated using the formula: $\mathrm{SSA}=\mathrm{SA}_{\text {part }} /\left(\mathrm{V}_{\text {part }} *\right.$ density $)$. Here $V_{\text {part }}$ is particle volume and $S_{\text {part }}$ is particle SA.

The calculated SSA uses the concept of maximally random jammed (MRJ) state [13], which is the most disordered state among all strictly jammed packing [14]. If the particles are in an MRJ state, then they cannot move or rattle around. The packing fraction for MRJ states ranges from 0.52 to

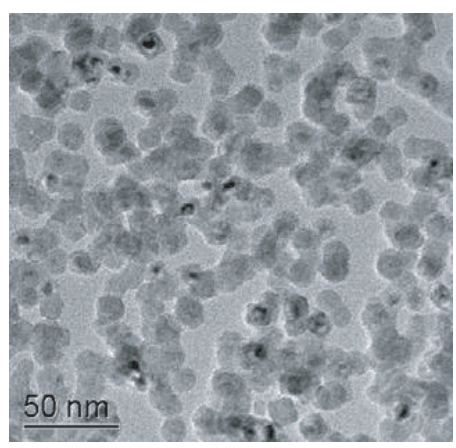

(a)

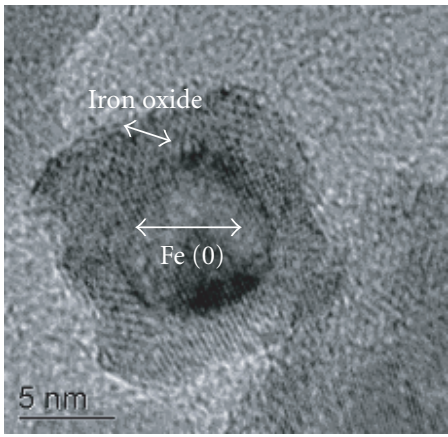

(b)

FIGURE 2: (a) Low-resolution TEM image of iron-iron oxide coreshell nanoclusters. (b) High-resolution TEM image of an iron-iron oxide core-shell nanocluster.

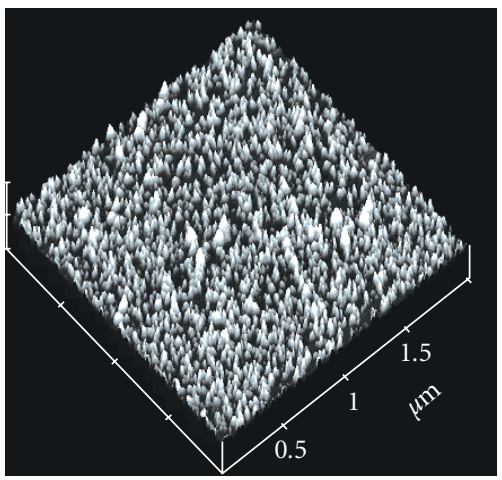

FIGURE 3: AFM image of iron-iron oxide core-shell nanoclusters ( $x$ $y: 2 \mu \mathrm{m}$ scale, $z=20 \mathrm{~nm}$, RMS $=1.98 \mathrm{~nm}$ ). 


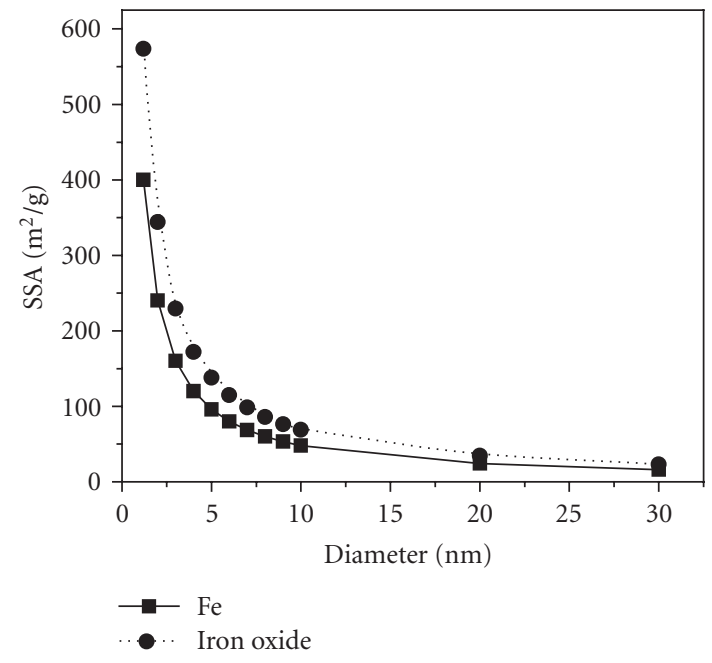

(a)

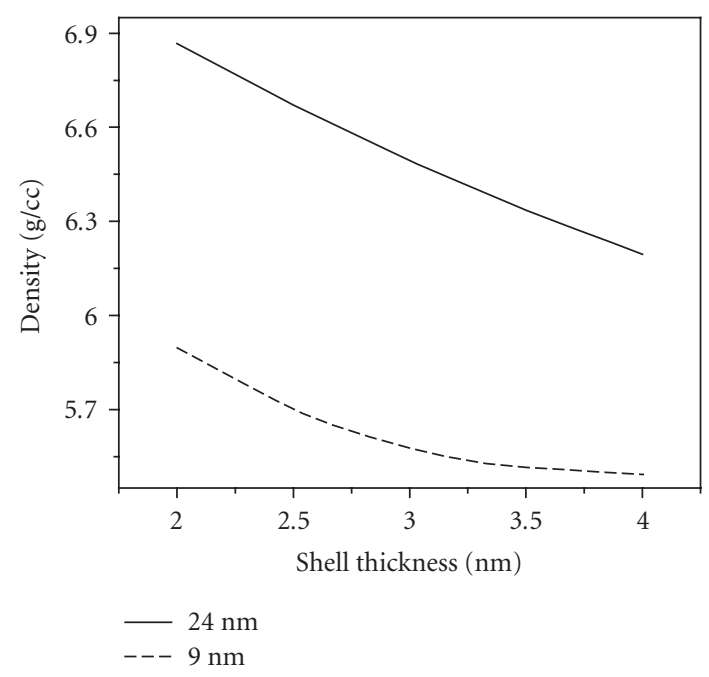

(b)

FIgURE 4: (a) Specific surface area (calculated) versus diameter curves for an iron and iron oxide particle film. (b) Density (bulk) versus oxide shell thickness of $9 \mathrm{~nm}$ and $24 \mathrm{~nm}$ core-shell iron-iron oxide samples.

0.74, but a packing fraction (PF) around 0.63 is much more common than others. PF increases with increasing order of configuration and lowers with the decrease in order [13].

Figure 4(a) shows the calculated SSA versus cluster diameter curves for iron and iron oxide porous cluster films. The particles are assumed spherical with bulk densities. Contact areas between particles are considered negligible. It is seen from Figure 4(a) that the SSA increases with the decrease in cluster diameter, and the increase is prominent below $10 \mathrm{~nm}$. From Figure 4(a), it is seen that iron and iron oxide nanoclusters show only a small change in SSA above $10 \mathrm{~nm}$ sizes. Below $5 \mathrm{~nm}$ diameter, the discrepancy between SSA of metallic iron and iron oxide increases.

Clusters $<7 \mathrm{~nm}$ in size, when observed under TEM, are found to be fully oxidized, while the clusters $>7 \mathrm{~nm}$ have an oxide shell of thickness range $2 \mathrm{~nm}$ to $3 \mathrm{~nm}$ [7]. For a cluster of size $21 \mathrm{~nm}$, the shell thickness can range from $2 \mathrm{~nm}$ to $3.5 \mathrm{~nm}$. If we consider a nanocluster to have the density of bulk $\mathrm{Fe}$ or/and iron oxide, then as the thickness of the shell of a nanocluster increases, its density tends towards the iron oxide density from the iron density. Figure 4(b) gives a plot of density versus shell thickness for $9 \mathrm{~nm}$ and $24 \mathrm{~nm}$ ironiron oxide nanoclusters. Density decreases faster in the case of $24 \mathrm{~nm}$ core-shell clusters with the increase in shell thickness, whereas the density decrease takes place at a slower pace for $9 \mathrm{~nm}$ core-shell clusters.

BET measures adsorption of nitrogen on the surface of a sample to determine the SSA of the particles. Here, BET is used to determine the SSA of core-shell iron-iron oxide nanoclusters of different sizes between $7 \mathrm{~nm}$ and $21 \mathrm{~nm}$ deposited on $\mathrm{Si}$ wafer substrates. Figure 5 shows BET results from the nanocluster film composed of spherical nanoclusters deposited on Si wafers, results calculated using the density of $24 \mathrm{~nm}$ clusters with $3 \mathrm{~nm}$ thick oxide shell as solid curve, and the BET results from plane Si wafers. Experimental results

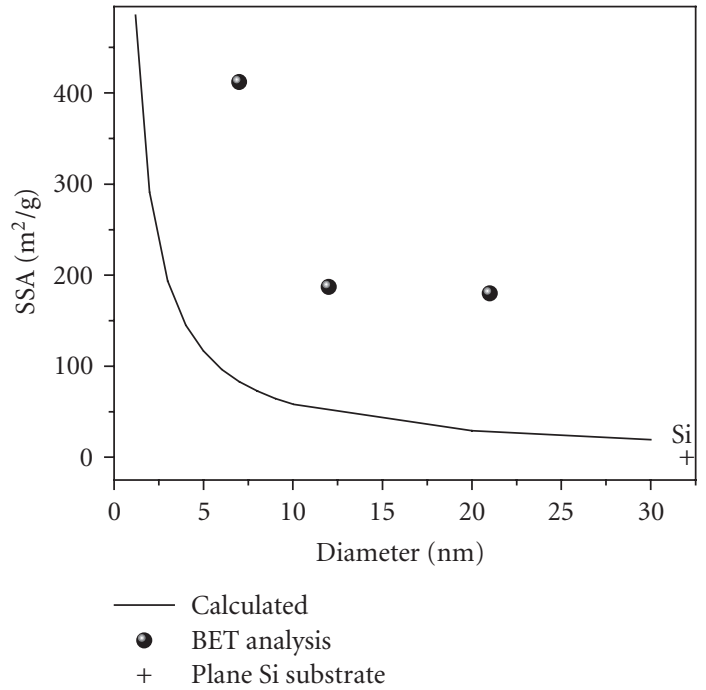

FIGURE 5: SSA (calculated) versus diameter curves for iron-iron oxide core-shell structured nanocluster with $3 \mathrm{~nm}$ shell thicknesses is denoted by the solid curve, SSA of nanoclusters deposited on Si wafers are given by spheres, and the SSA of plane Si wafers measured by BET is represented by plus sign.

have significantly large SSA than that expected from calculation, which can be due to lower nanocluster density and high porosity of the cluster film. Additionally, in the case of MRJ packing, particles are considered to be tightly packed, which is not strictly true for the soft landed nanoclusters deposited on Si wafer to form a thin film of clusters. This is because the nanoclusters that are deposited randomly leave lots of space in between each other and hence increases the amount of nitrogen absorbed into the sample. 


\section{CONCLUSIONS}

In conclusion, stable monodispersed iron-iron oxide coreshell nanoporous films are synthesized using nanoclusters as building blocks. TEM, AFM, and BET measurements are done to characterize this nanoporous sample. TEM image shows the monodispersed nanocluster sample. BET measurements show that the surface areas of cluster films are significantly enhanced with decrease in particle size.

\section{ACKNOWLEDGMENTS}

Research work performed in this paper is supported by the grant from US Department of Energy (DOE) Office of Science, Offices of Basic Energy Sciences, Biological, and Environmental Research. TEM, AFM, and BET measurements were done at Environmental Molecular Sciences Laboratory, a national scientific user facility sponsored by the DOE's Office of Biological and Environmental Research at PNNL. Idaho NSF-EPSCoR Grant provided funding and support for the synthesis capabilities for this work.

\section{REFERENCES}

[1] Y. Ding, M. Chen, and J. Erlebacher, "Metallic mesoporous nanocomposites for electrocatalysis," Journal of the American Chemical Society, vol. 126, no. 22, pp. 6876-6877, 2004.

[2] E. Schofield, "Reviews in modern surface finishing no.1: anodic routes to nanoporous materials," Transactions of the Institute of Metal Finishing, vol. 83, no. 1, pp. 35-42, 2005.

[3] J. T. Nurmi, P. G. Tratnyek, V. Sarathi, et al., "Characterization and properties of metallic iron nanoparticles: spectroscopy, electrochemistry, and kinetics," Environmental Science \& Technology, vol. 39, no. 5, pp. 1221-1230, 2005.

[4] W.-X. Zhang, "Nanoscale iron particles for environmental remediation: an overview," Journal of Nanoparticle Research, vol. 5, no. 3-4, pp. 323-332, 2003.

[5] Y. Q. Liu, S. A. Majetich, R. D. Tilton, D. S. Sholl, and G. V. Lowry, "TCE dechlorination rates, pathways, and efficiency of nanoscale iron particles with different properties," Environmental Science \& Technology, vol. 39, no. 5, pp. 1338-1345, 2005.

[6] Y. Qiang, J. Antony, M. G. Marino, and S. Pendyala, "Synthesis of core-shell nanoclusters with high magnetic moment for biomedical applications," IEEE Transactions on Magnetics, vol. 40, no. 6, pp. 3538-3540, 2004.

[7] J. Antony, Y. Qiang, D. R. Baer, and C. Wang, "Synthesis and characterization of stable iron-iron oxide core-shell nanoclusters for environmental applications," Journal of Nanoscience and Nanotechnology, vol. 6, no. 2, pp. 568-572, 2006.

[8] J. Antony, J. Nutting, D. R. Baer, and Y. Qiang, "Nanoporous and nanostructured materials made out of clusters for environmental applications," in Proceedings of the Materials Research Society Symposium (MRS '05), vol. 876E, p. R10.4, San Francisco, Calif, USA, 2005.

[9] J. Antony, S. Pendyala, A. Sharma, et al., "Room temperature ferromagnetic and ultraviolet optical properties of Co-doped $\mathrm{ZnO}$ nanocluster films," Journal of Applied Physics, vol. 97, no. 10, Article ID 10D307, 3 pages, 2005.

[10] Y. Qiang, R. F. Sabiryanov, S. S. Jaswal, Y. Liu, H. Haberland, and D. J. Sellmyer, "Magnetism of Co nanocluster films," Physical Review B, vol. 66, no. 6, Article ID 064404, 4 pages, 2002.
[11] Y. Qiang, Y. Thurner, T. Reiners, O. Rattunde, and H. Haberland, "Hard coatings $\left(\mathrm{TiN}, \mathrm{Ti}_{x} \mathrm{Al}_{1-x} \mathrm{~N}\right)$ deposited at room temperature by energetic cluster impact," Surface and Coatings Technology, vol. 100-101, pp. 27-32, 1998.

[12] D. J. Sellmyer, C. P. Luo, Y. Qiang, and J. P. Liu, "Magnetism of nanophase composite films," in Handbook of Thin Films Materials: Nanomaterials and Magnetic Thin Films, vol. 5, chapter 7, pp. 337-374, Academy press, New York, NY, USA, 2002.

[13] A. R. Kansal, S. Torquato, and F. H. Stillinger, "Diversity of order and densities in jammed hard-particle packings," Physical Review E, vol. 66, no. 4, Article ID 041109, 8 pages, 2002.

[14] A. Donev, F. H. Stillinger, and S. Torquato, "Unexpected density fluctuations in jammed disordered sphere packings," Physical Review Letters, vol. 95, no. 9, Article ID 090604, 2005. 

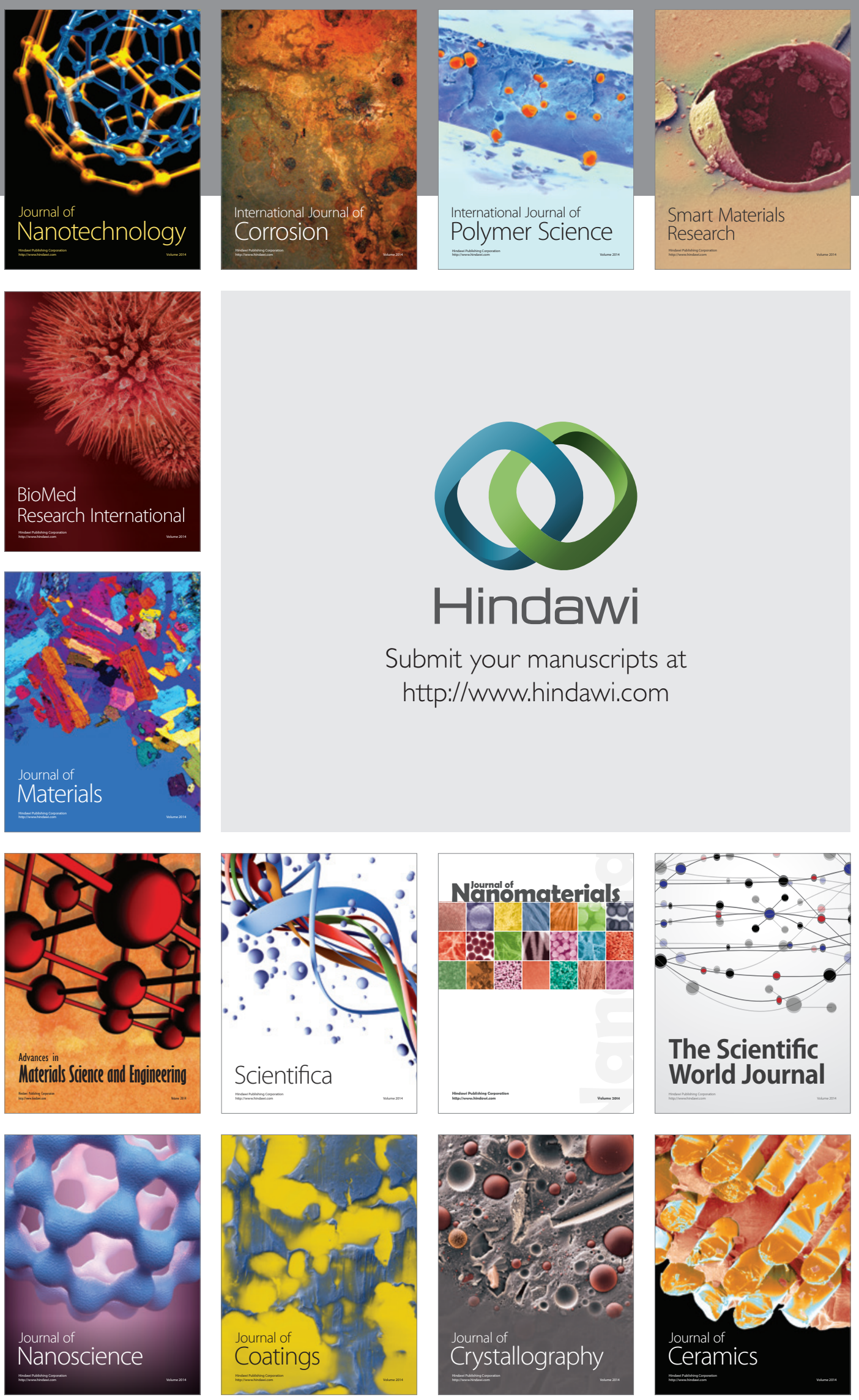

The Scientific World Journal

Submit your manuscripts at

http://www.hindawi.com

\section{World Journal}

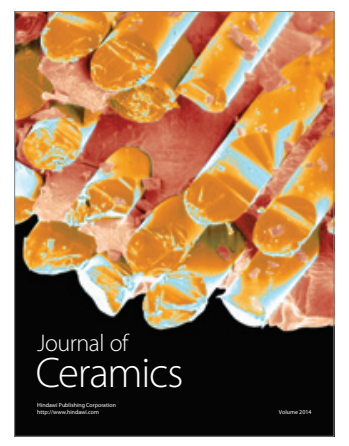

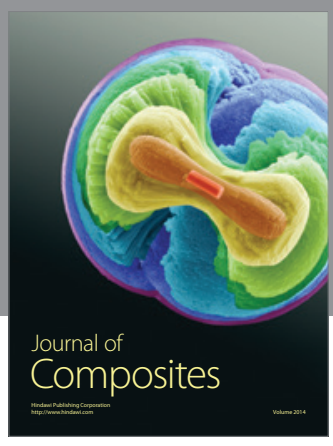
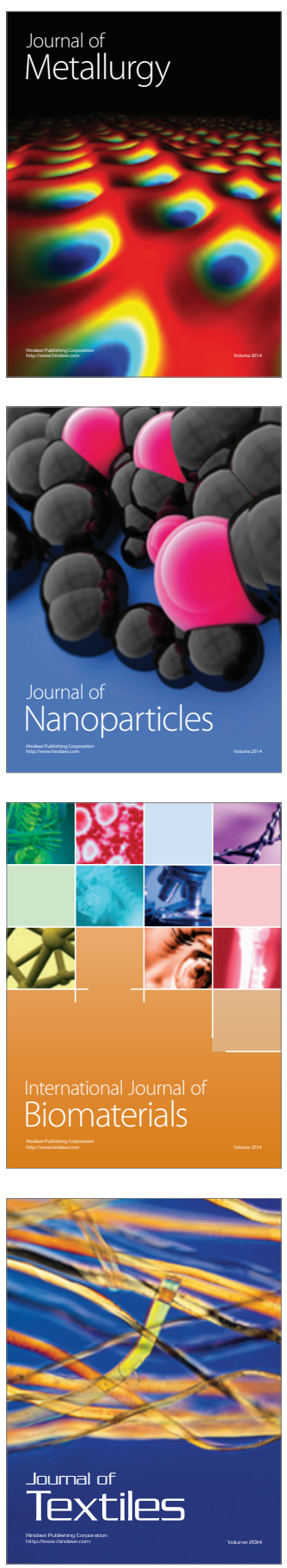